\title{
Basiliximab in pediatric liver transplantation: A pharmacokinetic-derived dosing algorithm
}

\author{
Kovarik JM, Gridelli BG, Martin S, Rodeck B, Melter M, Dunn SP, \\ Merion RM, Tzakis AG, Alonso E, Bucuvalas J, Sharp H, Gerbeau C, \\ Chodoff L, Korn A, Hall M. Basiliximab in pediatric liver \\ transplantation: A pharmacokinetic-derived dosing algorithm.
}

Pediatr Transplantation 2002: 6: 224-230. (C) 2002 Blackwell Munksgaard

Abstract: The pharmacokinetics and immunodynamics of basiliximab were assessed in 37 pediatric de novo liver allograft recipients to rationally design a dose regimen for this agegroup. In part one of the study, patients were given $12 \mathrm{mg} / \mathrm{m}^{2}$ basiliximab by bolus intravenous injection after organ perfusion and on day 4 after transplant. An interim pharmacokinetic evaluation supported a fixed-dose approach for part two of the study in which infants and children received two 10-mg doses of basiliximab and adolescents received two 20-mg doses. Blood samples were collected over a 12-week period for screening for antiidiotype antibodies and analysis of basiliximab and soluble interleukin-2 receptor (IL-2R) concentrations. Basiliximab clearance in infants and children $<9$ yr of age $(n=30)$ was reduced by $\approx 50 \%$ compared with adults from a previous study and was independent of age to $9 \mathrm{yr}$, weight to $30 \mathrm{~kg}$, and body surface area to $1.0 \mathrm{~m}^{2}$. Clearance in children and adolescents 9-14 $\mathrm{yr}$ of age $(\mathrm{n}=7)$ approached or reached adult values. An average of $15 \%$ of the dose was eliminated via drained ascites fluid, and drug clearance via this route averaged $29 \%$ of total body clearance. Patients with $>5 \mathrm{~L}$ of ascites fluid drainage tended to have lower systemic exposure to basiliximab. CD25-saturating basiliximab concentrations were maintained for $27 \pm 9$ days in part one of the study $\left(\mathrm{mg} / \mathrm{m}^{2}\right.$ dosing) with infants exhibiting the lowest durations. CD25 saturation lasted $37 \pm 11$ days in part two of the study, based on the fixeddose regimen ( $p=0.004 \mathrm{vs} . \mathrm{mg} / \mathrm{mg}^{2}$ dosing), but did not show the age-related bias observed in part one of the study. Anti-idiotype antibodies were detected in four patients, but this did not influence the clearance of basiliximab or duration of CD25 saturation. All 40 enrolled patients were included in the intent-to-treat clinical analysis. Episodes of acute rejection occurred in 22 patients $(55 \%)$ during the first 12 months post-transplant. Three patients experienced loss of their graft as a result of technical complications, and six patients died during the 12-month study. Basiliximab was well tolerated by intravenous bolus injection, with no cytokine-release syndrome or other infusion-related adverse events. Hence, basiliximab was safe and well tolerated in pediatric patients undergoing orthotopic liver transplantation. To achieve similar basiliximab exposure as is efficacious in adults, pediatric patients $<35 \mathrm{~kg}$ in weight should receive two $10-\mathrm{mg}$ doses and those $\geq 35 \mathrm{~kg}$ should receive two 20 -mg doses of basiliximab by intravenous infusion or bolus injection. The first dose should be given within $6 \mathrm{~h}$ after organ perfusion and the second on day 4 after transplantation. A supplemental dose may be considered for patients with a large volume of drained ascites fluid relative to body size.

\author{
John M. Kovarik', Bruno G. Gridelli ${ }^{2}$, \\ Steven Martin ${ }^{3}$, Burkhard Rodeck ${ }^{4}$, \\ Michael Melter", Stephen P. Dunn', \\ Robert M. Merion ${ }^{6}$, Andreas G. \\ Tzakis $^{7}$, Estella Alonso ${ }^{8}$, John \\ Bucuvalas ${ }^{9}$, Harvey Sharp ${ }^{10}$, \\ Christophe Gerbeau', Lawrence \\ Chodoff', Alexander Korn ${ }^{1}$ and \\ Michael Hall'
}

${ }^{1}$ Novartis Pharmaceuticals, Basel, Switzerland and East Hanover, USA, ${ }^{2}$ Ospedali Riuniti di Bergamo, Bergamo, Italy, ${ }^{3}$ Hôpital Sainte-Justine, Montreal, Quebec, Canada, ${ }^{4}$ Medizinische Hochschule Hannover, Germany, ${ }^{5}$ St. Christopher's Hospital for Children, Philadelphia, Pennsylvania, USA,

${ }^{6}$ University of Michigan Health System, Ann Arbor, Michigan, USA, ${ }^{7}$ University of Miami School of Medicine, Miami, Florida, USA, ${ }^{8}$ Children's Memorial Hospital, Chicago, Illinois, USA,

${ }^{9}$ Children's Hospital Medical Center, Cincinnati, Ohio, USA, ${ }^{10}$ University of Minnesota Hospital, Minneapolis, Minnesota, USA

Key words: basiliximab - pharmacokinetics - liver transplantation - pediatrics -

immunosuppression - monoclonal antibody cyclosporin A

John Kovarik, Novartis Pharma AG, Building WSJ 27.4093, 4002 Basel, Switzerland

Tel.: +41 613248239

Fax: +41 613242959

E-mail: john.kovarik@pharma.novartis.com

Accepted for publication 29 November 2001
Basiliximab is a high-affinity chimeric monoclonal antibody directed to the interleukin-2 receptor

\begin{abstract}
Abbreviations: AUC, area under the concentration-time curve; CD25, cluster of differentiation for IL-2 receptor, $\alpha$-chain; CL, total body clearance; CLasc, clearance via ascites fluid drainage; $\mathrm{C}_{\max }$., maximum concentration; CMV, cytomegalovirus; CsA, cyclosporin A; ELISA, enzyme-linked immunosorbent assay; IL-2R, interleukin-2 receptor; sIL-2R, soluble IL-2 receptor; $t_{1} / 2$, half-life; Vc, central distribution volume; Vss, steady-state distribution volume.
\end{abstract}

This work was presented, in part, at the American Society of Transplantion Congress 2000 and at the First Congress of the International Pediatric Transplant Association 2000. on activated T lymphocytes (IL-2R or CD25) and which is used to prevent acute cellular rejection in organ transplantation. A multi-center clinical trial has established the efficacy of basiliximab in adult liver transplantation (1). Basiliximab is applied in this indication in a two-dose regimen in the first 4 days after transplantation and provides CD25 saturation for an average of 4-6 weeks (2).

It is a reasonable premise that pediatric liver allograft recipients would also benefit from this agent. To assess this under controlled conditions, a multi-center, open-label study was performed to gain clinical experience with basiliximab in this 
population of patients while gathering pharmacokinetic and pharmacodynamic data to rationally develop a dosing algorithm across this age-group. This article presents the pharmacokinetic and pharmacodynamic aspects of the study with a summary of the main clinical findings.

The specific objectives were as follows:

1 To characterize the pharmacokinetics of basiliximab in pediatric de novo liver allograft recipients and explore for demographic covariates such as age, weight, and body surface area.

2 To characterize the pharmacodynamics of basiliximab by serial soluble IL-2R measurements in serum.

3 To screen for anti-idiotype antibody responses.

4 To use the composite of the above evaluations to derive a dosing algorithm for the rational use of basiliximab in pediatric liver transplant patients.

5 To assess the safety, tolerability, and preliminary efficacy of basiliximab, in combination with CsA microemulsion $\left(\right.$ Neoral ${ }^{\mathbb{R}}$, Novartis Pharmaceuticals, Basel, Switzerland) and corticosteroids, for the prevention of acute rejection episodes.

\section{Patients and methods}

\section{Study design}

This multi-center, open-label study enrolled 40 pediatric de novo liver transplant patients. Boys and girls under $16 \mathrm{yr}$ of age undergoing a first liver transplant from a cadaveric or living donor were eligible. Patients with fulminant hepatic failure, hemodynamic instability requiring pressor and/or inotropic support, primary non-function of the allograft, or graft thrombosis requiring re-operation or retransplantation, were excluded. Basiliximab was added to a baseline $\mathrm{CsA}$ /corticosteroid immunosuppressive regimen. Target CsA trough levels were $150-500 \mathrm{ng} / \mathrm{mL}$ in month 1 and $150-300 \mathrm{ng} / \mathrm{mL}$ thereafter.

The study was divided into two parts. In part one, 20 patients were enrolled and received a basiliximab regimen scaled down from the adult dose according to body surface area. An interim pharmacokinetic evaluation was then performed to design a rational dose regimen to be prospectively evaluated. In part two of the study, 20 patients were enrolled and received a stepwise fixed-dose regimen with a weight transition point as described below. Patients in both parts of the study were followed-up for 12 months to assess the safety and tolerability of basiliximab in this population of patients and to evaluate acute rejection episodes and graft and patient survival at 6 and 12 months post-transplantation. The Institutional Review Board or Research Ethics Committee at each participating institution approved the protocol, and each patient's parent or guardian gave written, informed consent.

\section{Drug formulation and dosing}

Basiliximab (Simulect ${ }^{\circledR}$, Novartis Pharmaceuticals) was supplied as a lyophilizate of $10-\mathrm{mg}$ strength. Immediately prior to administration, lyophilizates were reconstituted in $2.5 \mathrm{~mL}$ of sterile water for injection. In part one of the study, a body surface area-adjusted dosing approach was applied. Patients were administered two doses of basiliximab, $12 \mathrm{mg} / \mathrm{m}^{2}$ (not to exceed $20 \mathrm{mg}$ ), each. In part two of the study, patients who weighed $<40 \mathrm{~kg}$ received two doses of $10 \mathrm{mg}$ and those who weighed $\geq 40 \mathrm{~kg}$ received two doses of $20 \mathrm{mg}$. Doses were administered by bolus intravenous injection in $\approx 10 \mathrm{~s}$. The first dose was injected within $8 \mathrm{~h}$ after organ perfusion on day 0 ; the second dose was administered 4 days after transplant surgery. Basiliximab was given in addition to CsA microemulsion and a standard corticosteroid taper.

\section{Pharmacokinetic sampling}

Eighteen 1-mL venous blood samples were obtained over a 12-week period after transplantation. Specifically, samples were drawn before and $30 \mathrm{~min}$ after each dose of basiliximab (on days 0 and 4), daily through day 7 , and then in weeks $2,3,4,5,6,8,10$, and 12. Blood samples were drawn into collection tubes without anti-coagulants and allowed to clot at ambient temperature. Serum was harvested within $2-3 \mathrm{~h}$ of blood collection and frozen at $-18^{\circ} \mathrm{C}$ at the local laboratory until shipment to the central analytical site. Total volumes of drained ascites fluid were recorded daily while the drain was in place to a maximum of day 14. A 5-mL sample of each daily total volume was centrifuged, following which $3 \mathrm{~mL}$ of the supernatant was transferred to a plastic tube and frozen at $-18^{\circ} \mathrm{C}$.

\section{Basiliximab serum concentrations}

Basiliximab concentrations were measured in serum and ascites fluid with a validated idiotype-specific two-step competitive ELISA method using a fluorescein-labeled polyclonal rabbit anti-idiotype antibody and biotinylated basiliximab, as previously described (3). Assay accuracy ( $\%$ deviation from the nominal concentration) for quality control samples ranged from $-2.6 \%$ to $0.6 \%$ and assay precision ranged from 5.0 to $6.8 \%$. The quantification limit was $0.06 \mu \mathrm{g} / \mathrm{mL}$ for both serum and ascites fluid.

\section{SIL-2R concentrations}

sIL-2R serum concentrations were measured using a commercially available ELISA kit (Boehringer Mannheim Biochemie, Mannheim, Germany). Because basiliximab complexes with sIL-2R rendering it undetectable by the probe monoclonal antibodies in the kit, serum samples were pretreated by $1: 20$ dilution in assay buffer and addition of $25 \mu \mathrm{g} / \mathrm{mL}$ anti-idiotype antibody to dissociate the basiliximabsIL-2R complex, which allowed detection of sIL-2R. Following this step, the assay was then performed according to the manufacturer's instructions. Assay accuracy for quality control samples ranged from -0.7 to $1.4 \%$ and precision ranged from 6.7 to $9.3 \%$. The quantification limit was $11 \mathrm{ng} / \mathrm{mL}$.

\section{Anti-idiotype antibody screening}

Serum samples from baseline and week 12 were screened for antiidiotype antibodies with a validated competitive ELISA method in which a fixed amount of fluorescein-labeled rabbit anti-idiotype antibody and the human anti-idiotype antibody possibly present in the sample compete for binding with a fixed amount of biotinylated basiliximab. The quantification limit was $0.3 \mu \mathrm{g} / \mathrm{mL}$.

\section{Pharmacokinetic evaluation}

A standard two-compartment pharmacokinetic model was fitted to the basiliximab concentration-time data to derive the following pharmacokinetic parameters: $C_{\max }$. after the first bolus injection, cumulative $A U C, \mathrm{CL}, \mathrm{Vc}$ and Vss, and elimination $t_{1 / 2}$. The CLasc 


\section{Kovarik et al.}

was calculated as the quotient of the total amount of basiliximab eliminated via this route divided by the corresponding $A U C$ in serum.

\section{Duration of CD25 saturation}

The duration of CD25 saturation was estimated by two metrics. The pharmacokinetic metric was the duration post-transplant over which serum basiliximab concentrations exceeding $0.1 \mu \mathrm{g} / \mathrm{mL}$ (the in vivo threshold saturating concentration determined in adult patients) (3). The sIL-2R metric was taken from the sIL-2R profiles as the time post-transplant when the sIL-2R concentration decreased by $\geq 10 \%$ from the plateau phase maximum.

\section{Safety and efficacy}

Safety and tolerability were evaluated by recording laboratory variables, physical examinations, and vital signs, and by the incidence of adverse events (particularly those related to immunosuppression such as viral, fungal or opportunistic infections and malignant neoplasms), serious adverse events and infections. Efficacy was evaluated by recording the incidence of acute rejection episodes within 12 months after transplantation and actual patient and graft survival at 12 months after transplantation. An acute rejection was defined to have occurred if the rejection episode was biopsy confirmed and anti-rejection treatment administered.

\section{Results}

Demographics

Forty patients were enrolled in the study. Two patients did not receive the second dose and one did not receive any basiliximab. The 37 patients evaluable for pharmacokinetics were 15 boys and 22 girls categorized as 20 infants $(<2 \mathrm{yr}), 15$ children (2-11 yr), and two adolescents (12-14 yr). They ranged in age from 2 months to $14 \mathrm{yr}$, in weight from 4 to $44 \mathrm{~kg}$, and in body surface area from 0.23 to $1.38 \mathrm{~m}^{2}$. There were 30 whites, three blacks, and four of other ethnicities. The majority of patients $(n=33)$ received an organ from a cadaveric donor.

\section{Study course and dosing strategies}

Eighteen evaluable patients were enrolled in part one of the study and received the adult dose scaled down by body surface area: namely, $12 \mathrm{mg} / \mathrm{m}^{2}$ on days 0 and 4 . This corresponded to $7.7 \pm 4.2 \mathrm{mg} /$ dose (range: $3.6-17.0 \mathrm{mg}$ ). The protocol-scheduled interim pharmacokinetic evaluation indicated that basiliximab clearance in infants and children was reduced compared with adults but independent of age, weight, and body surface area. Clearance in adolescents began to take on or reach adult values. On this basis, a stepwise, fixeddose regimen with a weight transition point was selected for part two of the study. Specifically, infants and children received two 10-mg doses and adolescents received two 20 -mg doses (the standard adult regimen). The $40-\mathrm{kg}$ transition point between the two dosage regimens was chosen based on data available at the interim evaluation. Part two of the study enrolled 19 evaluable patients.

\section{Pharmacokinetics}

The average $C_{\max }$ in part two of the study (fixeddose regimen) was $5.6 \pm 1.4 \mu \mathrm{g} / \mathrm{mL}$ (range, 3.4-9.0) and did not differ significantly from study part one $\left(\mathrm{mg} / \mathrm{m}^{2}\right.$ dosing): $6.1 \pm 1.9 \mu \mathrm{g} / \mathrm{mL}$ (range, 3.5-9.7), $\mathrm{p}=0.44$. Likewise, $A U C$ values were similar with the two dosing approaches: $34 \pm 13 \mu \mathrm{g} \cdot \mathrm{day} / \mathrm{mL}$ in part one and $35 \pm 12 \mu \mathrm{g} \cdot$ day/ $\mathrm{mL}$ in part two $(\mathrm{p}=0.74)$. As clearance, distribution volume, and $t_{1 / 2}$ are dose-independent parameters, these data were pooled across all patients from the total study population $(n=37)$ to examine their relationship to demographic characteristics.

\section{Demographic covariates}

The relationships of clearance vs. age and weight are shown in Fig. 1. The relationship to body surface area was similar to that of weight (data not shown). The plots indicated that for infants and children, clearance was independent of age (up to $9 \mathrm{yr}$ ) and weight (up to $\approx 30 \mathrm{~kg}$ ). This was confirmed by linear regression analysis inasmuch as the slopes of the regression lines through these data were not significantly different from zero: $0.468 \mathrm{~mL} / \mathrm{h} / \mathrm{yr} \quad(\mathrm{p}=0.38)$ and $0.414 \mathrm{~mL} / \mathrm{h} / \mathrm{kg}$ $(p=0.06)$. Clearance in older children and adolescents 9-14 yr was transitional, with values starting to take on or reach adult values. These findings in the full population confirmed the clearance-demographic relationships characterized at the interim evaluation, as mentioned above. Clearance and other pharmacokinetic parameters are summarized in Table 1 and compared with adult values.

The Vc increased with age, weight, and body surface area over the full population range, as indicated by regression analyses showing signifi-

Table 1. Pharmacokinetics in pediatric and adult patients

\begin{tabular}{lccr}
\hline & $\begin{array}{c}\text { Infants/children } \\
<9 \mathrm{yr} \\
(\mathrm{n}=30)\end{array}$ & $\begin{array}{c}\text { Children/adolescents } \\
9-14 \mathrm{yr} \\
(\mathrm{n}=7)\end{array}$ & $\begin{array}{c}\text { Adults } \\
20-68 \mathrm{yr} \\
(\mathrm{n}=184)\end{array}$ \\
\hline Parameter & $19.3 \pm 6.5$ & $46.6 \pm 19.3$ & $55.0 \pm 26.2$ \\
$\mathrm{CL}(\mathrm{mL} / \mathrm{h})$ & $2.0 \pm 0.9$ & $4.0 \pm 1.3$ & $5.7 \pm 0.9$ \\
$V \mathrm{C}(\mathrm{L})$ & $4.1 \pm 3.9$ & $6.4 \pm 2.4$ & $9.7 \pm 4.2$ \\
$V$ Ss $(\mathrm{L})$ & $7.6 \pm 4.6$ & $5.8 \pm 2.5$ & $8.7 \pm 6.7$ \\
$t_{1 / 2}$ (days) & & &
\end{tabular}

$\mathrm{CL}$, clearance; $t_{1 / 2}$, half-life; $\mathrm{V}_{\mathrm{c}}$, central distribution volume; Vss, steady-state distribution volume.

Adult data were obtained from ref. 2 

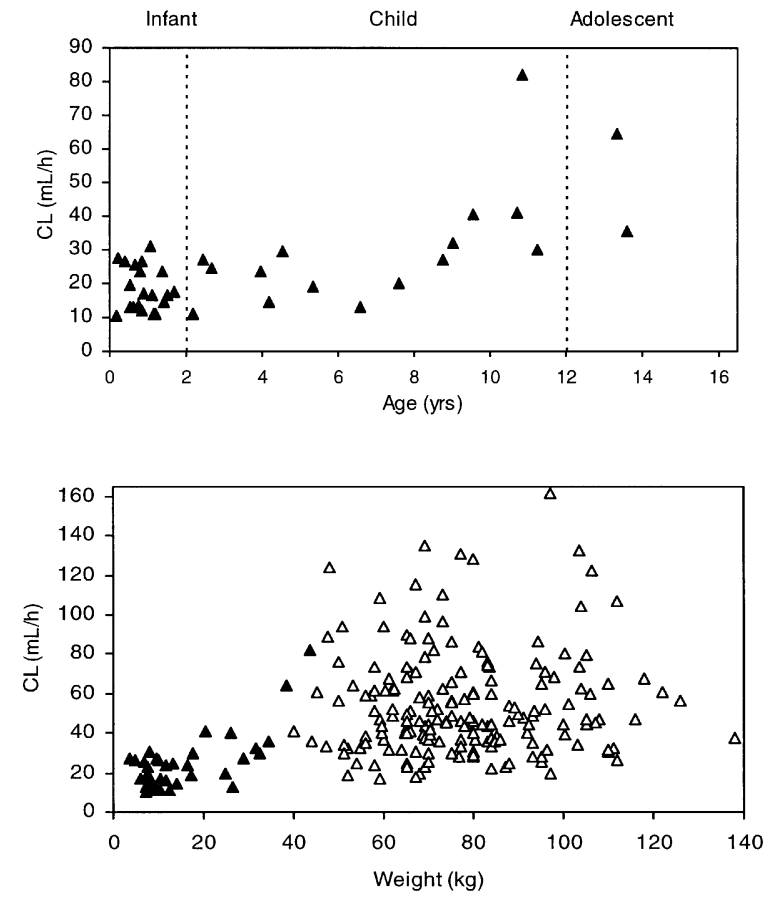

Fig. 1. Relationship between basiliximab clearance vs. age in pediatric patients in the upper panel and vs. weight in pediatric (filled triangle) and adult (open triangle) patients in the lower panel.

cant, positive slopes $(\mathrm{p}<0.001)$. The Vss was independent of age and body size. Both volume terms began to reach adult values in adolescence. The clinical impact of the dependence of $\mathrm{Vc}$ on body size applies mostly to the peak concentration whereby higher peaks would be expected in young, small patients when receiving the fixeddose regimen. This, however, was not the case (as noted above) as the $C_{\max }$. was similar among patients who received body surface area-based and fixed dosing. This was because the slopes of the relationships between $\mathrm{Vc}$ and age $(0.19 \mathrm{~L} / \mathrm{yr})$, weight $(0.07 \mathrm{~L} / \mathrm{kg})$, and body surface area $(2.35 \mathrm{~L} /$ $\mathrm{m}^{2}$ ) were very shallow. The elimination $t_{1 / 2}$ showed no dependence on age, weight, or body surface area among pediatric patients. Pediatric $t_{1 / 2}$ values were similar to those of adults, as shown in Table 1.

\section{Ascites fluid drainage}

Ascites fluid drains were in place for a median of 9 days (range: 3-36 days). Eight patients retained their drain beyond day 14 (range: 15-36 days). The median cumulative volume of drained fluid over the first 2 weeks after transplantation was $1.3 \mathrm{~L}$ (range: $0.2-28.8 \mathrm{~L}$ ). Cumulative drainages of $\leq 5 \mathrm{~L}$ had no discernible influence on the total body clearance of basiliximab, whereas patients with $>5 \mathrm{~L}$ of drainage $(\mathrm{n}=5)$ had generally higher total body clearances (median, $41.1 \mathrm{~mL} / \mathrm{h}$; range: $25.7-82.1 \mathrm{~mL} / \mathrm{h})$.

Ascites fluid samples for bioanalysis were received from 35 patients. With few exceptions, basiliximab was quantifiable in all samples. The median cumulative amount of basiliximab eliminated via drained fluid was $2.86 \mathrm{mg}$ (range: $0.34-19.05 \mathrm{mg}$ ). This represented a median of $15.1 \%$ (range: $1.7-67.1 \%$ ) of the total basiliximab dose administered. While the drain was in place, the median clearance via this route was $6.0 \mathrm{~mL} / \mathrm{h}$ (range: $0.7-50.1 \mathrm{~mL} / \mathrm{h}$ ) which represented, on average, $28.5 \%$ (range: $4.9-96.0 \%$ ) of the total body clearance.

\section{SIL-2R profiles}

Figure 2 shows a representative plot demonstrating the relationship between basiliximab pharmacokinetics and the time course of sIL-2R levels. sIL-2R measurements were performed in all patients $(n=18)$ in part one of the study. Before transplantation, sIL-2R concentrations were generally below or at the assay quantification limit $(11 \mathrm{ng} / \mathrm{mL})$. Inspection of the individual plots revealed a temporal pattern, whereby concentrations rose over the first 2-3 weeks after transplantation and then entered a plateau phase. Concentrations on day 4 prior to the second dose were $119 \pm 62 \mathrm{ng} / \mathrm{mL}$ and the average $C_{\max }$. plateau was $198 \pm 73 \mathrm{ng} / \mathrm{mL}$. After serum basiliximab concentrations reached the CD25 saturation threshold of $0.1 \mu \mathrm{g} / \mathrm{mL}$, sIL-2R concentrations began a precipitous decline, returning to baseline levels within 1-2 weeks, as demonstrated in Fig. 2. As blood samples for sIL-2R measurement were taken at weekly or bi-weekly intervals, a precise estimate of the number of days of CD25 saturation could not be derived with this metric. However, the time interval during which the decline from the plateau phase began was used as an estimate of the onset of CD25 de-saturation. This sIL-2R-guided estimate either included (44\% of profiles) or was within a median of 1 week $(56 \%$ of profiles) of the day on which serum basiliximab concentrations fell below $0.1 \mu \mathrm{g} / \mathrm{mL}$. Hence, this biomarker provided independent support for the pharmacokinetic estimate of the duration of CD25 saturation. The latter, as described below, was the primary metric for estimating the duration of receptor saturation.

\section{Duration of CD25 saturation}

Serum basiliximab concentrations on day 4 prior to administration of the second dose were $0.9 \pm 0.5$ $\mu \mathrm{g} / \mathrm{mL}$ (range: $0.3-1.8$ ). Regardless of the dosing 


\section{Kovarik et al.}

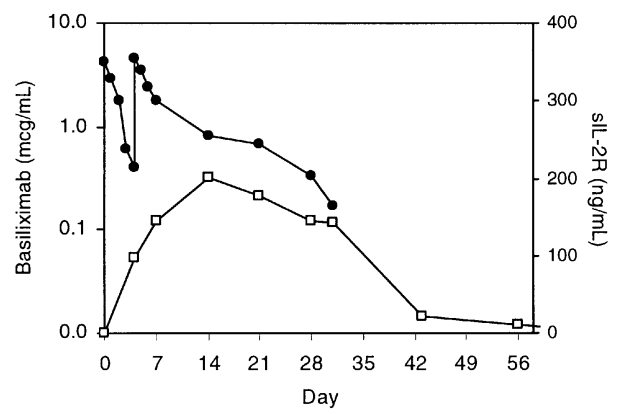

Fig. 2. Pharmacokinetic-dynamic profile from a representative pediatric patient showing the time courses of basiliximab concentrations (filled circle) and soluble interleukin-2 receptor (IL-2R) levels (open square).

approach, CD25-saturating concentrations were maintained throughout the inter-dose interval. The overall duration of CD25-saturating concentrations in part one of the study was $27 \pm 9$ days (range: 13-46) with the lowest values among infants and young children, as shown in the upper panel of Fig. 3. With the fixed-dose approach of part two of the study, CD25 saturation lasted $37 \pm 11$ days (range: $21-62$ days; $p=0.004$ ), and did not exhibit an age-related bias. Figure 3 also demonstrates that the CD25 saturation durations from part two of the study conformed to the adult distribution, with the majority falling within adult quartiles 2 through 4 .

The enhanced clearance of basiliximab in patients with pronounced ascites fluid loss also had an impact on the duration of CD25 saturation, as shown in the lower panel of Fig. 3. As noted above for clearance, patients with ascites fluid drainage in excess of $5 \mathrm{~L}$ in the first posttransplant week tended to have CD25 saturation duration in the lower adult distribution quartiles.

\section{Anti-idiotype antibody responses}

Of the 39 patients enrolled who received one or both basiliximab doses, three had no serum samples available after transplantation that were free of quantifiable basiliximab and therefore these samples could not be screened as a result of potential assay interference. Of the remaining 36 patients, four $(11 \%)$ had detectable anti-idiotype antibody ranging from 0.5 to $1.7 \mu \mathrm{g} / \mathrm{mL}$. Despite the presence of anti-idiotype antibody, the clearance of basiliximab (range: $13-26 \mathrm{~mL} / \mathrm{h}$ ) and the duration of CD25 saturation (range: 23-62 days) did not appear to be affected.

\section{Refinement of the pediatric dosing algorithm}

The appropriateness of the $40-\mathrm{kg}$ weight transition point used in part two of the study could not be examined in detail as few older children and
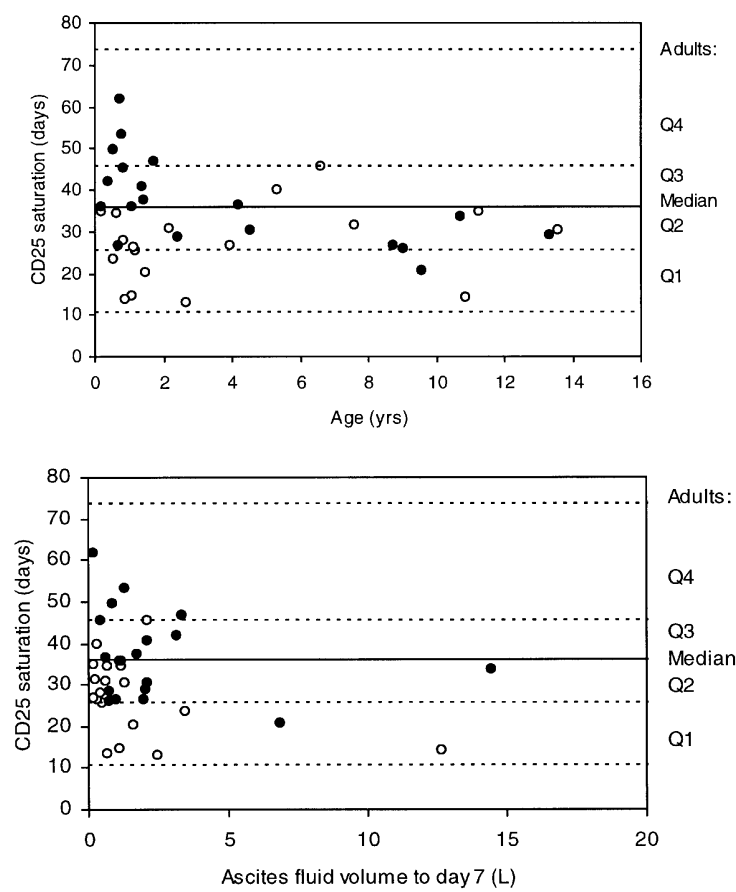

Fig. 3. Duration of CD25 saturation vs. age (upper panel) and drained ascites fluid volume (lower panel) from part one of the study with $\mathrm{mg} / \mathrm{m}^{2}$ dosing (open circle) and from part two of the study with fixed dosing (filled circle). Gridlines identify the distribution quartiles $(\mathrm{Q})$ from adults.

adolescents around this weight point were enrolled. Data from a similar study in pediatric kidney allograft patients (4) were combined, as shown in Fig. 4. A weight transition point of $35 \mathrm{~kg}$, as used in renal transplantation (4), also conformed to the clearance-weight pattern from pediatric liver transplantation. With the combined data set, selection of $35 \mathrm{~kg}$ for transferring between the two pediatric dose regimens appeared to be more appropriate than $40 \mathrm{~kg}$.

\section{Clinical findings}

The combined sample of patients from both parts of the study were separated into two age cohorts

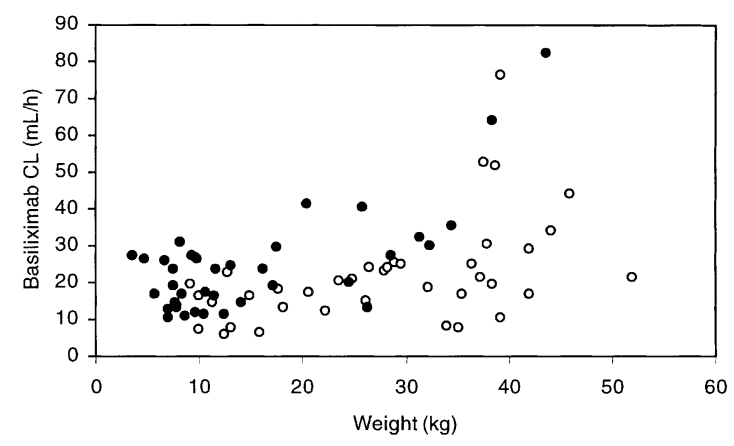

Fig. 4. Comparison of the clearance-weight relationships of basiliximab in pediatric liver (filled circle) and kidney (open circle) transplant patients. 
( $<3 \mathrm{yr}$ and $3-15 \mathrm{yr}$ ) for analyses of clinical outcomes. Acute rejection occurred in $55 \%$ of patients and was slightly higher $(60 \%)$ for $3-15-y r-$ old patients than for those $<3 \mathrm{yr}$ of age $(52 \%)$. The incidence of rejection was higher than in studies of basiliximab in adult liver transplant patients, but is similar to the incidence reported for other immunosuppressive regimens in pediatric patients (5-7). Approximately half of these rejections occurred while $\mathrm{CD} 25$ was saturated with the remaining half occurring after desaturation. The duration of CD25 saturation in patients with a rejection episode was similar to that of patients who remained rejection free. Three patients $(7.5 \%)$ experienced loss of their first graft as a result of technical complications, and six patients $(15 \%)$ died during the study.

All patients experienced adverse events, the incidence and distribution of which conformed to the expected profile of liver transplant recipients. The most common adverse events were hypertension $(70 \%)$, fever $(50 \%)$, and diarrhea $(50 \%) ; 25 \%$ of adverse events were considered to be related to the study medication. One patient developed malignancy (post-transplant lymphoproliferative disorder in month 9 post-transplant). Infections were experienced by 36 patients $(90 \%)$. Most reported infections for which an organism was identified were bacterial ( $58 \%$ of patients) or viral (35\% of patients). The most frequently reported manifestation of infection was sepsis $(30 \%$ of patients). The protocol recommended CMV prophylaxis with the final decision left to the clinician. CMV infections were reported in 10 patients $(25 \%)$. Basiliximab was well tolerated by intravenous bolus injection, without evidence of cytokine-release syndrome, acute hypersensitivity or other infusion-related adverse events. There were six deaths, all of which occurred in patients $<3 \mathrm{yr}$ of age, and none were caused by rejection.

\section{Discussion}

This multi-center study has gathered extensive pharmacokinetic data, along with tolerability and safety information, on the use of basiliximab in pediatric de novo liver transplant patients. Importantly, the population was well distributed over the full demographic ranges for age ( 2 months to $14 \mathrm{yr}$ ), weight (4-44 $\mathrm{kg}$ ), and body surface area $\left(0.23-1.38 \mathrm{~m}^{2}\right)$, which are representative of those in the general pediatric liver transplant population. The two-part study design and the data it generated provide support for a rational dosing algorithm for pediatric liver allograft recipients.
Part one of the study, using a $\mathrm{mg} / \mathrm{m}^{2}$ dosing regimen scaled down from that employed in adult liver transplantation, indicated that basiliximab clearance in infants and young children was relatively independent of the common demographic variables conventionally employed in pediatric dosing, namely: age, weight, and body surface area. On average, clearance in this agerange was reduced by $\approx 50 \%$ compared with adults. On this basis, a fixed-dose approach was applied in part two of the study, whereby infants and children received one-half of the standard adult dose, specifically, two 10-mg doses. Clearances in older children and adolescents were either intermediate between values in younger children and adults or were similar to adult values. On this basis, they received the standard adult dose of two 20-mg doses in part two of the study. The transition point between the two regimens was based on weight, with $40 \mathrm{~kg}$ appearing to separate the two populations adequately, based on data from part one of the study.

It was the intention in part two of the study to provide confirmation of these clearance-demographic relationships in a greater number of patients, as well as to focus additional attention on the transition point between childhood and adolescence, which was sparsely represented in part one. The complete study results (from a total of 30 infants and children ranging in age from 2 months to 9 yr) fully supported the clearancedemographic relationship described above, from part one of the study, that clearance is independent of common demographic covariates. At the transition between childhood and adolescence $(10-14 \mathrm{yr})$ there were, however, only five patients in the full study population. These data alone did not allow a specific weight cut-off to be specified for transferring between the two regimens. When these data in liver-transplanted pediatric patients were supplemented with those from a similar study in pediatric kidney transplantation (4), they were in agreement with the approach taken in the latter transplant indication to use $35 \mathrm{~kg}$ as the weight transition point, as shown in Fig. 4.

Loss of basiliximab in ascites fluid drainage was demonstrable in all patients in this study and agrees with similar observations in adult liver allograft recipients (3). Because duration of ascites drainage and cumulative volume are highly patient-specific, this route of drug elimination can inflate the overall pharmacokinetic variability in $\mathrm{CL}$ and distribution volume across the population. When cumulative volumes were $\leq 5 \mathrm{~L}$ (which is usually the case in uncomplicated 


\section{Kovarik et al.}

post-transplant courses) there was no major impact on overall clearance. Total volumes in excess of $5 \mathrm{~L}$ tended to be associated with higher total body clearances and a shorter duration of CD25 saturation. Combining these data with the experience in adult liver transplantation (2) suggested that a supplemental dose in these cases may be considered on a case-by-case basis.

The patterns noted for drug clearance were paralleled by similar patterns in the duration of CD25 saturation. In part one of the study, when dosing was based on body surface area, infants and very young children had the shortest duration of saturation compared with the remainder of the pediatric population and with the adult distribution of values. This was a direct consequence of the fact that infants and very young children had the same drug clearance as older children but received lower milligram doses because of a smaller body size. With the fixed-dose regimen in part two of the study, the pattern was improved for this age cohort, as shown with the filled symbols in Fig. 3. Moreover, the distribution of duration of CD25 saturation was more uniform across the full pediatric demographic range and better centered on that achieved in adults.

Basiliximab had a low potential for immunogenicity in this pediatric population. Four patients had anti-idiotype antibody responses. Nonetheless, these did not appear to be neutralizing antibodies inasmuch as the basiliximab clearances and duration of CD25 saturation in these patients were similar to values in the remainder of the study population.

The three main pharmacokinetic conclusions from this study which support a rational basiliximab dosing algorithm for pediatric de novo liver transplantation were:

1 Basiliximab clearance in infants and children was reduced by $\approx 50 \%$ compared with adults but was independent of age, weight, and body surface area. Clearance among adolescents was similar to that of adults.

2 Body surface area-adjusted dosing yielded a shorter duration of CD25 saturation in infants and very young children compared with older pediatric patients and adults. The fixed-dose approach used in part two of the study resolved this problem

3 Loss of basiliximab in drained ascites fluid contributed to a higher CL and shorter duration of CD25 saturation, especially when the total drained volume exceeded $\approx 5 \mathrm{~L}$ in the first week after transplantation.

On the basis of these data and those from a similar study in pediatric kidney transplantation (4), a fixed dose of $10 \mathrm{mg}$ administered within $6 \mathrm{~h}$ after organ perfusion and again on day 4 after transplantation is recommended for pediatric patients who weigh $<35 \mathrm{~kg}$. For pediatric patients who weigh $\geq 35 \mathrm{~kg}$, the standard adult dose is recommended, namely, two 20-mg doses. An additional dose of 10 or $20 \mathrm{mg}$, respectively, on day 7 may be considered on a case-by-case basis, depending on the volume of ascites fluid drained relative to the patient's body size. Our data indicate that a drainage of $>5 \mathrm{~L}$ in the first week after transplantation should alert the clinician to consider an additional dose.

\section{References}

1. Neuhaus P, Nashan B, Clavien PA, et al. Basiliximab (Simulect) reduces the rate and severity of acute rejection in adult liver transplant recipients. Transplantation 2000: 69: S118.

2. Kovarik JM, Nashan B, Neuhaus $P$, et al. A population screen to identify demographic-clinical covariates of basiliximab in liver transplantation. Clin Pharmacol Ther 2001: 69: 201-209.

3. Kovarik JM, Breidenbach T, Gerbeau C, korn A, Schmidt AG, Nashan B. Disposition and immunodynamics of basiliximab in liver allograft recipients. Clin Pharmacol Ther 1998: 64: 66-72.

4. Offner G, Broyer M, Loirat C, et al. Disposition of basiliximab in de novo pediatric renal transplantation. Pediatr Nephrol 1999: 13: C24.

5. Eckhoff DE, D'Allessandro AM, Knechtle SJ, et al. 100 consecutive liver transplants in infants and children: An 8-year experience. J Pediatr Surg 1994: 29: 1135-1139.

6. Vazquez J, Gamez M, Santamaria ML, et al. Liver transplantation in small babies. J Pediatr Surg 1993: 28: 1051- 1053.

7. Cacciarelli TV, Esquivel CO, Cox KL, et al. Oral tacrolimus (FK506) induction therapy in pediatric orthotopic liver transplantation. Transplantation 1996: 61: 1188-1192. 